\title{
EDITORIAL
}

\section{Focus on foot and ankle}

\author{
Thomas Mittlmeier ${ }^{1}$
}

(c) The Author(s) 2021

Dear Reader,

Ankle fractures represent common injuries. Correspondingly, the treatment of ankle fractures is routine in hospitals of any level of care. For decades standard radiographic techniques were employed as the sole diagnostic tool and traditional nonoperative and surgical strategies according to AO/OTA recommendations at that time were believed to suffice for a generally favourable outcome [1]. An even cursory view at the contemporary literature reveals on the one side that there is a lack of studies with high evidence despite the high incidence of ankle fractures and on the other side that the long-term outcome in ankle fractures is at best moderate $[2,3]$. Involvement of the syndesmotic and/or the delta ligament complex and bi-, tri- and quadrimalleolar fracture types have a remarkably poor prognosis, in particular, if anatomic reduction of any fracture component and restoration of ligamentous stability are missed [4]. With the advent and consecutive spread of spatial analysis employing CT scanning the detection of hidden fracture components, the understanding of the injury pattern of the ankle mortise and the relevance of anatomic reduction have considerably increased [5]. Consequently, this lead to the postulate of a postoperative CT control as an appropriate measure to confirm the quality of reduction in complex fracture patterns. Further, the spatial analysis promoted a better comprehension of the biomechanical dimensions of distinct fracture components, e.g., the posterior malleolus, encompassing a paradigm shift and modified surgical techniques [4].

The intraoperative application of cone beam CT in cases with a complex fracture pattern and unstable mortise allowed for substituting the postoperative $\mathrm{CT}$ by intraoperative assessment of adequate reduction and eventual correction

Thomas Mittlmeier

thomas.mittlmeier@med.uni-rostock.de

1 Department of Trauma, Hand and Reconstructive Surgery, Rostock University Medical Center, Rostock, Germany of non-anatomic reduction during the same procedure rendering a second-step surgical revision unnecessary [6].

Until now, fibular rotation and maltorsion of the fibula after ankle fracture with concomitant lesion of the syndesmotic complex has gained limited attendance in the literature, only. Adequate determination of fibular rotation is one of three topics of research provided by Sven Vetter et al. [7]. Their group from Ludwigshafen/Germany was one of the first worldwide to study intraoperative 3-D geometry of the foot and ankle joint via cone beam CT [6]. In the first manuscript of our focus edition they studied 100 healthy ankle joints to define the most appropriate location to measure fibular rotation. They now recommend to assess fibular rotation at a level of $6 \mathrm{~mm}$ below the ankle joint line with the utmost reliability and reproducibility compared with neighboring alternative locations for measurement [7].

Their second paper [8] addresses the intraoperative use of cone beam CT to evaluate anatomic reduction in ankle fractures with unstable syndesmotic injuries. At a mean followup of 6 years they found inferior outcomes and a higher rate of posttraumatic osteoarthritis in patients with incomplete syndesmotic reduction compared with those with anatomic reduction. In the third publication from the Ludwigshafen group a cadaver study had been performed employing 22 cadaver legs where Vetter et al. [9] dissected the anterior part of the syndesmotic complex, the interosseous membrane and, additionally, made an osteotomy of the posterior malleolus. They found that in the unloaded situation corresponding to the intraoperative condition neither the release of the syndesmosis nor the osteotomy of the posterior malleolus led to a manifest fibular malposition detectable during cone beam CT. Therefore, they hint at potentially unstable syndesmotic lesions despite non-pathologic morphology in cone beam CT examinations which emphasizes the additional need for intraoperative provocation tests as the application of a rotational test or the Cotton/hook test.

Michal Tuček and colleagues examined the clinical outcome following surgical treatment of ankle fractures with a large posterior malleolar fragment corresponding to a Bartoníček/Rammelt type 4 fracture [10]. A CT scan was 
available before and after surgical reconstruction demonstrating anatomic reconstruction in nine patients, only. Less than 3 years after surgery the patients with an anatomic reduction of the posterior malleolus exhibited the best functional results assessed via the AOFAS hindfoot score. Two out of six patients with posttraumatic osteoarthritis revealed either a malrotation of the fibula, a step-off at the joint line after reduction of the posterior malleolus or a widening of the syndesmosis.

Finally, Bi and colleagues examined the diagnostic value of the intraoperative tap test for acute deltoid ligament instability which had been proposed by Rajagopalan et al. [12]. In a patient collective with 92 ankle fractures they compared the tap test versus the gravity stress test and performed an open dissection of the deltoid ligament [11]. In particular, the negative predictive value of the tap test with $100 \%$ is superior to the gravity stress test with $95.6 \%$.

In summary, the manuscripts from our current focus topic underline the notion that the treatment of ankle fracture is rapidly further developing towards a more predictable outcome. I do hope that the lecture facilitates the integration of these findings into your daily work.

Funding Open Access funding enabled and organized by Projekt DEAL.

\section{Declarations}

Conflict of interest The author declares that he has no conflict of interest.

Open Access This article is licensed under a Creative Commons Attribution 4.0 International License, which permits use, sharing, adaptation, distribution and reproduction in any medium or format, as long as you give appropriate credit to the original author(s) and the source, provide a link to the Creative Commons licence, and indicate if changes were made. The images or other third party material in this article are included in the article's Creative Commons licence, unless indicated otherwise in a credit line to the material. If material is not included in the article's Creative Commons licence and your intended use is not permitted by statutory regulation or exceeds the permitted use, you will need to obtain permission directly from the copyright holder. To view a copy of this licence, visit http://creativecommons.org/licenses/by/4.0/.

\section{References}

1. Rammelt S, Swords M, Dhillon M, Sands A (eds). Manual of fracture management. Foot and ankle. Stuttgart, New York: Thieme, AO Foundation; 2020.

2. Stufkens SAS, van den Bekerom MPJ, Kerkhoffs GMMJ, Hintermann B, van Dijk CN. Long-term outcome after 1822 operatively treated ankle fractures: a systematic review of the literature. Injury. 2011;42:119-27.

3. Chong HH, Hau MYT, Mishra P, Rai P, Mangwani J. Patient outcomes following ankle fracture fixation. Foot Ankle Int. 2021 (Epub ahead of print).

4. Rammelt S, Bartoníček J. Posterior malleolar fractures. A critical analysis review. JBJS Rev 2020;8:e19.00207.

5. Rammelt S, Boszczyk A. Computed tomography in the diagnosis and treatment of ankle fractures. JBJS Rev 2018;6:e7.

6. Franke J, von Recum J, Suda AJ, Grützner PA, Wendl K. Intraoperative three-dimensional imaging in the treatment of acute unstable syndesmotic injuries. J Bone Jt Surg Am;2012;94-A:1386-1390.

7. Vetter SY, Gassauer M, Uhlmann L, Swartman B, Schnetzke M, Keil H, Franke J, Grützner PA, Beisemann N. A standardized computed tomography measurement method for distal fibular rotation. Eur J Trauma Emerg Surg. 2021. https://doi.org/10.1007/ s00068-019-01120-6.

8. Vetter SY Euler J, Beisemann N, Swartman B, Keil H, Grützner PA, Franke J. Validation of radiological reduction criteria with intraoperative cone beam CT in unstable syndesmotic injuries. Eur J Trauma Emerg Surg. 2021. https://doi.org/10.1007/ s00068-020-01299-z.

9. Vetter SY, Palesche N, Beisemann N, Schnetzke M, Keil H, Kirsch J, Grützner PA, Franke J. Influence of syndesmotic injuries and posterior malleolar ankle fractures on fibula position in the ankle joint: a cadaveric study. Eur J Trauma Emerg Surg. 2021. https://link.springer.com/article/https://doi.org/10.1007/ s00068-019-01292-1.

10. Tuček M, Rammelt S, Kostlivý K, Bartoníček J. CT controlled results of direct reduction and fixation of posterior malleolus in ankle fractures. Eur J Trauma Emerg Surg. 2021. https://link. springer.com/article/https://doi.org/10.1007/s00068-020-01309-0.

11. Bi C, Kong D, Lin J, Wang Q, Wu K, Huang J. Diagnostic value of intraoperative tap test for acute deltoid ligament injury. Eur J Trauma Emerg Surg. 2021. https://link.springer.com/ article/https://doi.org/10.1007/s00068-019-01243-w.

12. Rajagopalan S, Upadhyay V, Taylor HP, Sangar A. New intraoperative technique for testing the distal tibiofibular syndesmosis. Ann R Coll Surg Engl. 2010;92:258. 